\title{
Análise de modelos probabilísticos de frequência para obtenção de equações IDF na cidade de Cunha-SP
}

\author{
Analysis of probabilistic frequency models to obtain IDF \\ equations in the city of Cunha-SP.
}

Data de entrada: 07/12/2016

Data de aprovação: 09/08/2017

\section{Resumo}

Para o dimensionamento de obras hidráulicas, é importante que sejam usadas equações IDF bem ajustadas a cada local. A formulação de uma IDF depende da adequação de uma função teórica de distribuição de probabilidade à série histórica de precipitação. Esse trabalho avalia a adequação de seis funções de distribuição de probabilidade (normal, log-normal, Pearson tipo III, log-Pearson tipo III, Gumbel Teórica, Gumbel Finita) aos dados de precipitações máximas anuais de 21 estações pluviométricas do município de Cunha-SP e de municípios vizinhos. A avaliação das distribuições foi baseada no teste de Kolmogorov-Smirnov, no P-valor e em análises visuais. Como resultados, obteve-se que a função Gumbel Finita é a que melhor representou valores extremos. As distribuições Pearson tipo III e log-Pearson tipo III foram as que apresentaram menores P-valores. As IDFs de 5 a 60 min são proporcionais às de 60 a 1.440 min. Palavras-chave: Equações IDF. Distribuições probabilísticas. Teste de aderência. Análises visuais.

\section{Abstract}

For the design of hydraulic works, it is important that welladjusted local IDF equations are used. The formulation of an IDF equation depends on the adequacy of a theoretical function of probability distribution to the historical series of precipitation. This work evaluates the adequacy of six functions of probability distribution (normal, log-normal, Pearson type III, log-Pearson type III, Theoretical Gumbel, Finite Gumbel) to the data of maximum annual rainfall of 21 rainfall stations of Cunha county-SP and surroundings. The evaluation of distributions was based on the Kolmogorov-Smirnov test, the P-value test, and on visual analysis. As results, it was obtained that the Finite Gumbel function is the one that best represented extreme values. Pearson type III and log-Pearson type III distributions were shown to have lower P-values. IDFs from 5 to 60 min are proportional to those of 60 at $1440 \mathrm{~min}$. Keywords: IDF equations. Probabilistic distributions. Adherence test. Visual analysis.

Maria Luíza Teófilo Gandini - Mestre e Doutoranda em Engenharia de Infraestrutura Aeronáutica (área de Infraestrutura Aeroportuária) pelo Instituto Tecnológico de Aeronáutica. Professora assistente da Universidade Federal de Ouro Preto.

Paulo Ivo Braga de Queiroz - Mestre em Engenharia de Infraestrutura Aeronáutica. Doutor em Engenharia Aeronáutica e Mecânica pelo Instituto Tecnológico de Aeronáutica. Professor adjunto do Instituto Tecnológico de Aeronáutica.

*Endereço para correspondência: Rua Barão de Ouro Branco, 99, Bairro Antônio Dias, Ouro Preto, MG.CEP: 35.4000-000. E-mail: mltgandiniagmail.com. 


\section{INTRODUÇÃO}

Chuva intensa é a ocorrência extrema de precipitação, com duração, distribuição temporal e espacial críticas para uma área ou bacia hidrográfica (BERTONI e TUCCI, 2015). Como a chuva é uma variável aleatória contínua, pode-se representá-la por meio de alguma distribuição teórica de probabilidade.

O estudo das relações intensidade-duração-frequência (IDF) das precipitações extremas é de grande interesse para os trabalhos de hidrologia, devido à frequente aplicação na estimativa das vazões de projeto para dimensionamento de obras de engenharia, tais como vertedores de barragens, bueiros, bocas de lobo, terraços, canais de drenagem (BACK, 2006).

\section{OBJETIVO}

Este trabalho teve como objetivo determinar qual distribuição de probabilidade, entre normal, log-normal, Pearson tipo III, log-Pearson tipo III, Gumbel Teórica e Gumbel Finita, melhor se adequava aos dados de precipitação diária de 21 estações pluviométricas do município de Cunha e seu entorno, que fazem parte da sub-bacia do Alto Vale do Paraíba do Sul, comparando as frequências amostrais com as frequências teóricas conjuntamente com diagnóstico gráfico e posteriormente avaliando os parâmetros das IDFs obtidas.

\section{METODOLOGIA}

A Tabela 1 reúne as informações acerca das estações escolhidas.

Tabela 1: Resumo das estações escolhidas e suas características (HIDROWEB, 2015).

\begin{tabular}{|c|c|c|c|c|c|c|}
\hline Id & Código & Nome & Operadora & Lat. & Long. & Altitude \\
\hline 3 & 2244014 & Capetinga & DAEE-SP & $-22^{\circ} 51^{\prime} 00^{\prime \prime}$ & $-44^{\circ} 47^{\prime} 00^{\prime \prime}$ & 1060 \\
\hline 4 & 2244015 & Campos de Cunha & DAEE-SP & $-22^{\circ} 55^{\prime} 00^{\prime \prime}$ & $-44^{\circ} 49^{\prime} 00^{\prime \prime}$ & 1020 \\
\hline 5 & 2244026 & Vargem do Tanque & DAEE-SP & $-22^{\circ} 56^{\prime} 00^{\prime \prime}$ & $-44^{\circ} 55^{\prime} 00^{\prime \prime}$ & 880 \\
\hline 6 & 2244048 & Campos de Cunha & CPRM & $-22^{\circ} 55^{\prime} 16^{\prime \prime}$ & $-44^{\circ} 49^{\prime} 20^{\prime \prime}$ & 750 \\
\hline 7 & 2244096 & Campos do Cunha & LIGHT & $-22^{\circ} 55^{\prime} 13^{\prime \prime}$ & $-44^{\circ} 49^{\prime} 13^{\prime \prime}$ & 1010 \\
\hline 8 & 2245055 & Estrada de Cunha & CPRM & $-22^{\circ} 59^{\prime} 45^{\prime \prime}$ & $-45^{\circ} 02^{\prime} 30^{\prime \prime}$ & 790 \\
\hline 9 & 2245056 & Estrada de Cunha (DA) & DAEE-SP & $-22^{\circ} 59^{\prime} 00^{\prime \prime}$ & $-45^{\circ} 30^{\prime} 00^{\prime \prime}$ & 800 \\
\hline 10 & 2245164 & Paraitinga & DAEE-SP & $-22^{\circ} 59^{\prime} 59^{\prime \prime}$ & $-45^{\circ} 03^{\prime} 00^{\prime \prime}$ & 790 \\
\hline 11 & 2344001 & Fazenda do Cume & DAEE-SP & $-23^{\circ} 05^{\prime} 00^{\prime \prime}$ & $-44^{\circ} 54^{\prime} 00^{\prime \prime}$ & 900 \\
\hline 12 & 2344002 & Cunha (INMET) & DAEE-SP & $-23^{\circ} 04^{\prime} 00^{\prime \prime}$ & $-44^{\circ} 57^{\prime} 00^{\prime \prime}$ & 970 \\
\hline 13 & 2344003 & Sertão do Rio Manso & DAEE-SP & $-23^{\circ} 07^{\prime} 00^{\prime \prime}$ & $-44^{\circ} 51^{\prime} 00^{\prime \prime}$ & 1460 \\
\hline 14 & 2344009 & Alto S. do Mar - B. Mato Limpo & CPRM & $-23^{\circ} 09^{\prime} 13^{\prime \prime}$ & $-44^{\circ} 51^{\prime} 32^{\prime \prime}$ & 1050 \\
\hline 16 & 2344012 & Bairro Paraibuna & DAEE-SP & $-23^{\circ} 11^{\prime} 00^{\prime \prime}$ & $-44^{\circ} 59^{\prime} 00^{\prime \prime}$ & 1120 \\
\hline 17 & 2344015 & Cunha & LIGHT & $-23^{\circ} 04^{\prime} 45^{\prime \prime}$ & $-44^{\circ} 57^{\prime} 32^{\prime \prime}$ & 940 \\
\hline 18 & 2344018 & Cunha (EC) & DAEE-SP & $-23^{\circ} 05^{\prime} 00^{\prime \prime}$ & $-44^{\circ} 58^{\prime} 00^{\prime \prime}$ & 980 \\
\hline 19 & 2345005 & Capivara & DAEE-SP & $-23^{\circ} 04^{\prime} 00^{\prime \prime}$ & $-45^{\circ} 04^{\prime} 00^{\prime \prime}$ & 800 \\
\hline 21 & 2345174 & Ferraz & DAEE-SP & $-23^{\circ} 10^{\prime} 00^{\prime \prime}$ & $-45^{\circ} 06^{\prime} 00^{\prime \prime}$ & 880 \\
\hline 22 & 2245059 & Rocinha & DAEE-SP & $-22^{\circ} 54^{\prime} 00^{\prime \prime}$ & $-45^{\circ} 05^{\prime} 00^{\prime \prime}$ & 1000 \\
\hline 23 & 2345006 & Faxinal & DAEE-SP & $-23^{\circ} 07^{\prime} 00^{\prime \prime}$ & $-45^{\circ} 12^{\prime} 00^{\prime \prime}$ & 840 \\
\hline 24 & 2345007 & Lagoinha & DAEE-SP & $-23^{\circ} 05^{\prime} 00^{\prime \prime}$ & $-45^{\circ} 12^{\prime} 00^{\prime \prime}$ & 910 \\
\hline 25 & 2345117 & Lagoinha & LIGHT & $-23^{\circ} 00^{\prime} 18^{\prime \prime}$ & $-45^{\circ} 11^{\prime} 31^{\prime \prime}$ & 900 \\
\hline
\end{tabular}

\subsection{Teste de aderência}

Para verificar a aderência de uma distribuição proposta à distribuição empírica dos valores amostrais, realizam-se os chamados testes de aderência, principalmente quando não se conhe- ce a priori a distribuição de probabilidades que descreve a população da qual se extraiu um certo conjunto de observações, situação que é muito frequente no âmbito das variáveis aleatórias hidrológicas (NAGHETTINI e PINTO, 2007). 
Para distribuições contínuas, o teste de Kolmogorov-Sminorv (K-S) geralmente é o que melhor atende aos objetivos. A partir do teste de aderência de $\mathrm{K}-\mathrm{S}$, é possível comparar dois conjuntos de dados sem a necessidade de que esses conjuntos estejam ajustados a uma função de distribuição conhecida. Em outras palavras, trata-se de um teste não paramétrico (PRESS et al., 2007).

A estatística $D$ de Kolmogorov-Smirnov foi obtida pelo valor máximo da diferença absoluta entre duas funções de distribuição cumulativas. Assim, para comparar um conjunto de dados $S_{N}(x)$ à uma função distribuição cumulativa $\mathrm{P}(\mathrm{x})$, a estatística K-S é definida pela Equação 1:

$$
D=\underset{-\infty<x<\infty}{\operatorname{má} x}\left|S_{N}(x)-P(x)\right|
$$

As funções densidade de probabilidade acumuladas foram calculadas de acordo com as fórmulas apresentadas por Naghettini e Pinto (2007) para cada uma das 6 distribuições.

Desse modo, no intuito de verificar qual delas se adequou mais à série de dados, calculou-se o $\mathrm{P}$-valor (definido mais adiante), e quanto menor ele for, melhor será a distribuição.

Após o cálculo do $\mathrm{D}_{\text {máx }}$ do teste $\mathrm{K}-\mathrm{S}$, determina-se o número de pontos efetivos $\mathrm{N}_{\mathrm{e}}$. No caso da comparação envolvendo uma distribuição contínua, tem-se que o número de pontos efetivo é igual ao número de pontos experimentais, isto é $\mathrm{N}_{\mathrm{e}}=\mathrm{N}$.

De acordo com Press et al. (2007), para o cálculo do $\mathrm{P}$-valor, define-se inicialmente a variável z por meio da Equação 2:

$$
\begin{aligned}
& \text { Probabilidade }(D>\text { observado })= \\
& Q_{K S}\left(\left[\sqrt{N_{e}}+0,12+0,11 / \sqrt{N_{e}}\right] D\right)
\end{aligned}
$$

onde utiliza-se $D_{\text {máx }}$ no lugar de $D$. O cálculo do P-valor faz-se então pela Equação 3:

$$
P_{K S}(z)=\frac{\sqrt{2 \pi}}{z} \sum_{j=1}^{\infty} \exp \left(-\frac{(2 j-1)^{2} \pi^{2}}{8 z^{2}}\right) .
$$

\subsection{Equações IDFs}

As variáveis que caracterizam as chuvas intensas são a duração, a intensidade e a frequência de ocorrência, que é o inverso do período de retorno; são as equações intensidade-duração-frequência (IDF), que relacionam essas três variáveis, a Equação 4 exemplifica um tipo de fórmula em que a IDF pode ser apresentada:

$i=\frac{K \cdot \operatorname{Tr}^{c}}{\left(m+t_{d}\right)^{n}}$

onde i é a intensidade da chuva intensa $(\mathrm{mm} / \mathrm{h}), \mathrm{t}_{\mathrm{d}}$ é o tempo de duração da chuva (min), Tr é o tempo de recorrência (anos) e $\underline{K}, \underline{c}, \underline{m}$ e $\underline{n}$ são parâmetros adimensionais obtidos do ajuste, que são determinados para cada local.

Primeiramente, buscaram-se as séries históricas do local e, feito isso, os registros foram organizados por anos hidrológicos (para o sudeste do Brasil, o ano hidrológico se inicia em outubro e termina em setembro do ano seguinte, ou seja, ele compreende o início do período chuvoso até o fim do período seco).

Selecionou-se de cada ano hidrológico a maior chuva diária. Feito isso, as mesmas foram organizadas em ordem decrescente. Optou-se por aplicar o teste de outliers de Grubbs e Beck (1972), com o propósito de garantir que valores extremos, muito altos ou muito baixos, não atrapalhassem os demais procedimentos estatísticos.

Determinadas as séries de chuvas máximas anuais por ano hidrológico, escolheu-se a distribuição probabilística que era capaz de representar o comportamento das chuvas extremas no local de estudo, baseando-se nos testes de aderência de Kolmogorov-Smirnov, nos $\mathrm{P}$-valores e em análise visual dos gráficos da Figura 1, frisando que se escolheu a distribuição que melhor garantiria uma boa extrapolação para os maiores valores de precipitação. 
Figura 1: Modelos de probabilidade para todas as estações levando em conta as 6 distribuições estatísticas.

Modelos de probabilidade (Est. 2244014)

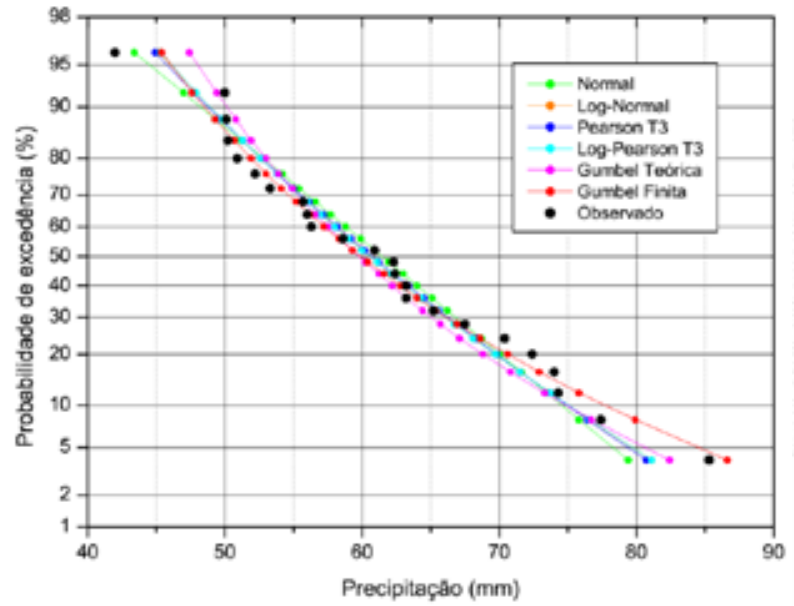

Modelos de probabilidade (Est. 2244026)


Modelos de probabilidade (Est. 2244015)

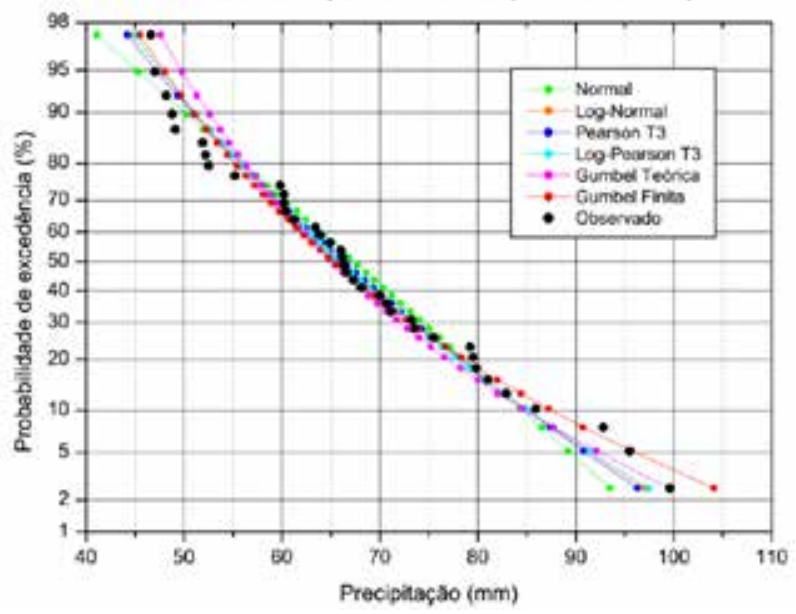

Modelos de probabilidade (Est. 2244048)

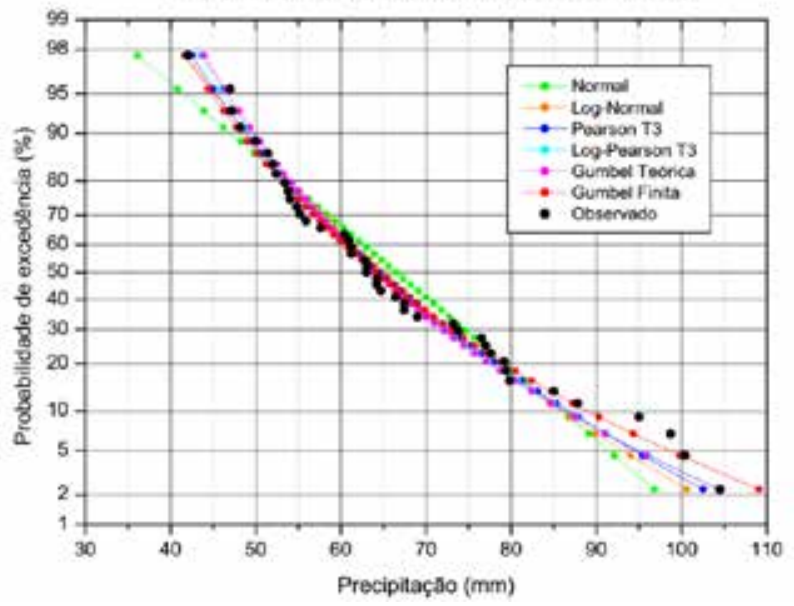

Modelos de probabilidade (Est. 2245055)




CONTINUAÇÃO Figura 1: Modelos de probabilidade para todas as estações levando em conta as 6 distribuições estatísticas.

CONTINUAÇÃO Figura 1: Modelos de probabilidade para todas as estações levando em conta as 6 distribuições estatísticas.
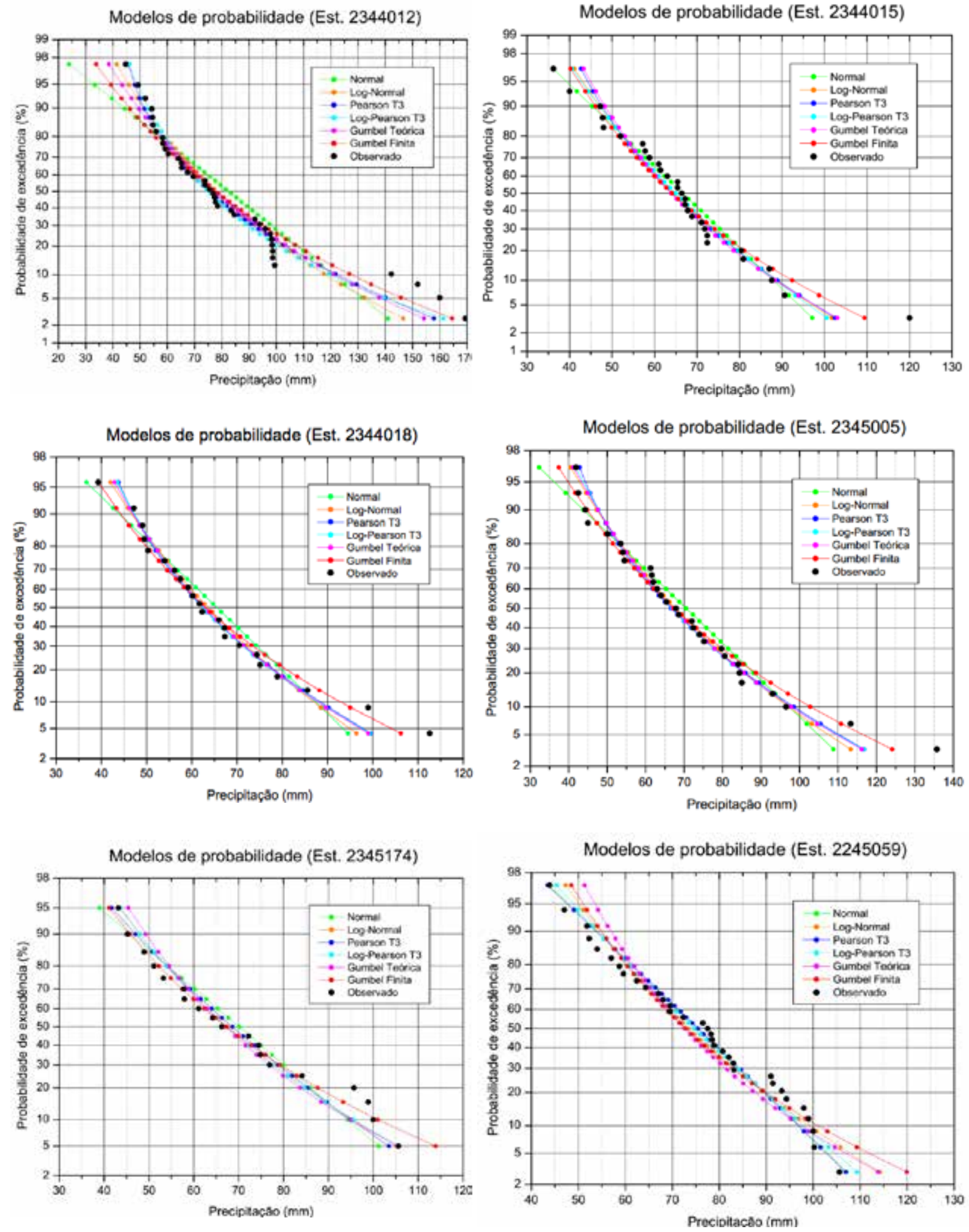
CONTINUAÇÃO Figura 1: Modelos de probabilidade para todas as estações levando em conta as 6 distribuições estatísticas.
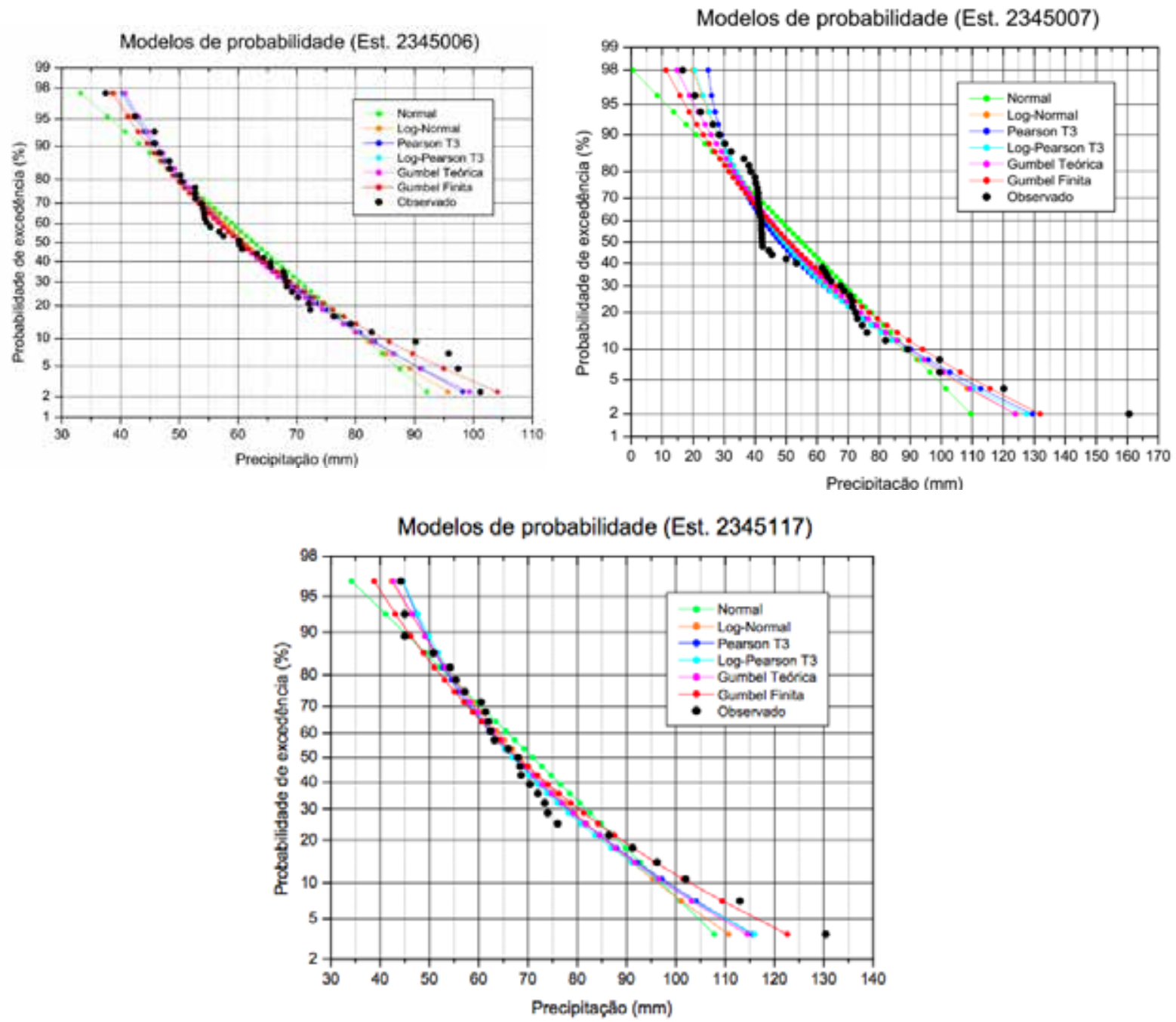

Uma vez escolhida a distribuição mais apropriada, utilizou-se a equação de Ven-Te-Chow (definida mais adiante) para obter as chuvas de 1 dia, referentes aos tempos de recorrência de 2, 5, 10, 20, 30, 50, 75 e 100 anos. Conforme Chow (1964), um quantil de uma variável hidrológica pode ser representado pela média $\bar{x}$ somada a um desvio $\Delta \mathrm{X}$ :

$X=\bar{x}+\Delta X$,

$\mathrm{O}$ termo $\Delta \mathrm{X}$ depende da dispersão característica da distribuição de $X$, do tempo de retorno e de outros parâmetros do modelo probabilístico. Ainda segun- do Chow (1964), o termo $\Delta \mathrm{X}$ pode ser assumido igual ao produto do desvio padrão $\sigma$ por um fator de frequência $K$, ou seja, $\Delta X=K \sigma$. O fator de frequência é uma função do tempo de retorno e da distribuição de probabilidades empregada na análise. Desse modo, a Equação 5 pode ser reescrita dando origem à Equação 6 (equação de Ven-Te-Chow):

$x_{T r}=\bar{x}+K \sigma$,

onde $\bar{x}$ é o valor médio da variável considerada, $\sigma$ é o desvio-padrão, e K é o fator de frequência. 
Utilizou-se o método dos coeficientes de desagregação com valores propostos pela Cetesb (1980) para o cálculo da altura de chuva para outras durações menores que 1 dia. Há de se ressaltar que primeiramente transformou-se a chuva de 1 dia em chuva de 24 horas, para depois obter-se as durações inferiores. A partir das alturas, chega-se às intensidades em milímetros por hora pela Equação 7:

$i=\frac{P}{t_{d}} \cdot 60$,

onde $P$ é a precipitação em milímetros e $t_{d}$ é $o$ tempo de duração da chuva em minutos.
Por meio dos três conjuntos dos valores de: períodos de retorno, durações e intensidades, fez-se uma regressão não linear, para obter os parâmetros da Equação 4.

\section{RESULTADOS}

A Tabela 3 reúne os valores de $D_{\text {máx }}$ para cada estação e distribuição estatística. Totalizaram-se 126 testes, dos quais apenas um apresentou rejeição para um valor de $\mathrm{D}$ crítico a $5 \%$, destacado em negrito e sublinhado nas Tabelas 2 e 3. O P-valor para cada estação e distribuição está apresentado na Tabela 3. Os espaços preenchidos com "-" devem-se ao fato de que os parâmetros da distribuição não se enquadravam no intervalo de validade, e por isso não foi possível fazer o cálculo de frequência.

Tabela 2: $D_{\text {máx }}$ do teste K-S para cada modelo probabilístico e a respectiva estação.

\begin{tabular}{|c|c|c|c|c|c|c|c|}
\hline ESTAÇÃO & $\mathbf{3}$ & $\mathbf{4}$ & $\mathbf{5}$ & $\mathbf{6}$ & $\mathbf{7}$ & $\mathbf{8}$ & $\mathbf{9}$ \\
\hline Normal & 0,104 & 0,081 & 0,103 & 0,128 & 0,153 & 0,135 & 0,136 \\
\hline Log-Normal & 0,088 & 0,082 & 0,095 & 0,086 & 0,111 & 0,078 & 0,095 \\
\hline Pearson & 0,092 & 0,078 & 0,087 & 0,075 & 0,109 & 0,068 & 0,123 \\
\hline Log-Pearson & 0,088 & 0,082 & - & 0,064 & 0,103 & 0,066 & - \\
\hline Gumbel Teórica & 0,104 & 0,105 & 0,132 & 0,069 & 0,086 & 0,070 & 0,102 \\
\hline Gumbel Finita & 0,105 & 0,102 & 0,130 & 0,069 & 0,110 & 0,082 & 0,096 \\
\hline ESTAÇÃO & $\mathbf{1 0}$ & $\mathbf{1 1}$ & $\mathbf{1 2}$ & $\mathbf{1 3}$ & $\mathbf{1 4}$ & $\mathbf{1 6}$ & $\mathbf{1 7}$ \\
\hline Normal & 0,166 & 0,110 & 0,154 & 0,143 & 0,114 & 0,183 & 0,160 \\
\hline Log-Normal & 0,116 & 0,078 & 0,113 & 0,127 & 0,068 & 0,124 & 0,119 \\
\hline Pearson & 0,118 & 0,092 & 0,111 & - & 0,063 & 0,126 & 0,118 \\
\hline Log-Pearson & 0,094 & 0,080 & 0,102 & - & 0,067 & 0,107 & - \\
\hline Gumbel Teórica & 0,112 & 0,083 & 0,108 & 0,150 & 0,074 & 0,135 & 0,126 \\
\hline Gumbel Finita & 0,158 & 0,109 & 0,143 & 0,119 & 0,149 & 0,160 & 0,130 \\
\hline ESTAÇÃO & $\mathbf{1 8}$ & $\mathbf{1 9}$ & $\mathbf{2 1}$ & $\mathbf{2 2}$ & $\mathbf{2 3}$ & $\mathbf{2 4}$ & $\mathbf{2 5}$ \\
\hline Normal & 0,143 & 0,110 & 0,117 & 0,092 & 0,118 & $\mathbf{0 , 2 1 1}$ & 0,184 \\
\hline Log-Normal & 0,093 & 0,087 & 0,107 & 0,114 & 0,097 & 0,169 & 0,127 \\
\hline Pearson & 0,068 & 0,114 & 0,110 & - & 0,077 & 0,144 & 0,111 \\
\hline Log-Pearson & 0,066 & 0,102 & 0,105 & - & 0,079 & 0,163 & 0,101 \\
\hline Gumbel Teórica & 0,072 & 0,099 & 0,112 & 0,142 & 0,081 & 0,172 & 0,116 \\
\hline Gumbel Finita & 0,111 & 0,111 & 0,082 & 0,127 & 0,076 & 0,161 & 0,140 \\
\hline
\end{tabular}

A média do $D_{\text {máx }}$ do teste $K-S$ em ordem crescente dá-se pela seguinte sequência: para a log-Pearson tipo III foi 0,070, para a Pearson tipo III foi 0,090, para a log-normal foi 0,104, para Gumbel Teórica foi 0,107, para a Gumbel Finita foi 0,118 e para a normal foi 0,135. 
Tabela 3: P-valor para cada modelo probabilístico e a respectiva estação.

\begin{tabular}{|c|c|c|c|c|c|c|c|}
\hline ESTAÇÃO & $\mathbf{3}$ & $\mathbf{4}$ & $\mathbf{5}$ & $\mathbf{6}$ & $\mathbf{7}$ & $\mathbf{8}$ & $\mathbf{9}$ \\
\hline Normal & 0,052 & 0,043 & 0,049 & 0,541 & 0,485 & 0,759 & 0,372 \\
\hline Log-Normal & 0,011 & 0,048 & 0,024 & 0,106 & 0,129 & 0,131 & 0,053 \\
\hline Pearson & 0,017 & 0,029 & 0,010 & 0,036 & 0,113 & 0,045 & 0,251 \\
\hline Log-Pearson & 0,011 & 0,050 & - & 0,007 & 0,075 & 0,037 & - \\
\hline Gumbel Teórica & 0,055 & 0,231 & 0,234 & 0,016 & 0,015 & 0,059 & 0,094 \\
\hline Gumbel Finita & 0,058 & 0,193 & 0,212 & 0,017 & 0,117 & 0,173 & 0,057 \\
\hline ESTAÇÃO & $\mathbf{1 0}$ & $\mathbf{1 1}$ & $\mathbf{1 2}$ & $\mathbf{1 3}$ & $\mathbf{1 4}$ & $\mathbf{1 6}$ & $\mathbf{1 7}$ \\
\hline Normal & 0,404 & 0,347 & 0,421 & 0,438 & 0,450 & 0,859 & 0,585 \\
\hline Log-Normal & 0,063 & 0,051 & 0,099 & 0,293 & 0,023 & 0,425 & 0,223 \\
\hline Pearson & 0,070 & 0,141 & 0,077 & - & 0,009 & 0,448 & 0,212 \\
\hline Log-Pearson & 0,008 & 0,066 & 0,046 & - & 0,019 & 0,249 & - \\
\hline Gumbel Teórica & 0,048 & 0,084 & 0,072 & 0,499 & 0,049 & 0,531 & 0,280 \\
\hline Gumbel Finita & 0,339 & 0,340 & 0,325 & 0,220 & 0,770 & 0,738 & 0,323 \\
\hline ESTAÇÃO & $\mathbf{1 8}$ & $\mathbf{1 9}$ & $\mathbf{2 1}$ & $\mathbf{2 2}$ & $\mathbf{2 3}$ & $\mathbf{2 4}$ & $\mathbf{2 5}$ \\
\hline Normal & 0,271 & 0,146 & 0,055 & 0,071 & 0,422 & $\mathbf{0 , 9 7 9}$ & 0,708 \\
\hline Log-Normal & 0,012 & 0,025 & 0,025 & 0,237 & 0,196 & 0,891 & 0,256 \\
\hline Pearson & 0,000 & 0,177 & 0,034 & - & 0,041 & 0,727 & 0,125 \\
\hline Log-Pearson & 0,000 & 0,093 & 0,022 & - & 0,054 & 0,865 & 0,067 \\
\hline Gumbel Teórica & 0,000 & 0,074 & 0,040 & 0,509 & 0,065 & 0,902 & 0,162 \\
\hline Gumbel Finita & 0,065 & 0,158 & 0,001 & 0,363 & 0,038 & 0,859 & 0,373 \\
\hline
\end{tabular}

A média do $\mathrm{P}$-valor em ordem crescente dá-se pela seguinte sequência: para a log-Pearson tipo III foi 0,104, para a Pearson tipo III foi 0,135, para a log-normal foi 0,158, para Gumbel Teórica foi 0,191, para a Gumbel Finita foi 0,273 e para a normal foi 0,403 .

Ressalta-se que se encontrou um detalhe relevante nesses testes ( $\mathrm{K}$-S e P-valor) para o caso particular das IDFs, que reside no fato de que desde as menores probabilidades às maiores são analisadas, ou seja, os parâmetros dos testes são obtidos por meio de análise de frequências teóricas e amostrais para todo o conjunto de dados. Contudo, para a construção de IDFs, deve-se atentar que o mais importante é o ajuste às menores probabilidade de excedência, a fim de que se permita fazer adequadas extrapolações com as equações obtidas, haja vista que para elaborar projetos de drenagem eficientes ao combate de danos provocados por cheias necessita-se de valores extremos de precipitação, de modo que para a construção da IDF é de suma importância que o modelo esco-
Ihido adira bem aos maiores valores de precipitação observados na série anual.

Para contornar essa particularidade e no intuito de observar o comportamento na parte final das distribuições, foi feita também uma análise visual (à qual deu-se grande peso) nos gráficos da Figura 1. A razão disso é que, caso seja feita a aplicação do teste K-S à totalidade da série, sem complementá-lo com a análise visual gráfica, o mesmo pode paliar a real tendência dos extremos máximos. Pode-se observar isso em um exemplo destacado em vermelho na Figura 2. No caso desta estação, para um nível de significância de $5 \%$, o parâmetro $D_{\text {crítico }}$ vale 0,198 e o $D_{\text {máx }}$ para log-Pearson tipo III e Pearson tipo III, respectivamente vale 0,067 e 0,063. Isso sugere que a segunda distribuição é mais representativa desse conjunto de dados. Todavia, ao avaliar a construção gráfica para essa estação, constata-se que a log-Pearson tipo III se aproxima mais dos extremos maiores; em vista disso, essa distribuição passa a ser mais indicada do que Pearson tipo III para extrapolação das precipitações máximas, nesse caso. 
Figura 2: Comparação entre as frequências observadas e as teóricas de cada modelo de probabilidade para a estação $2344009(\mathrm{Id}=14)$.

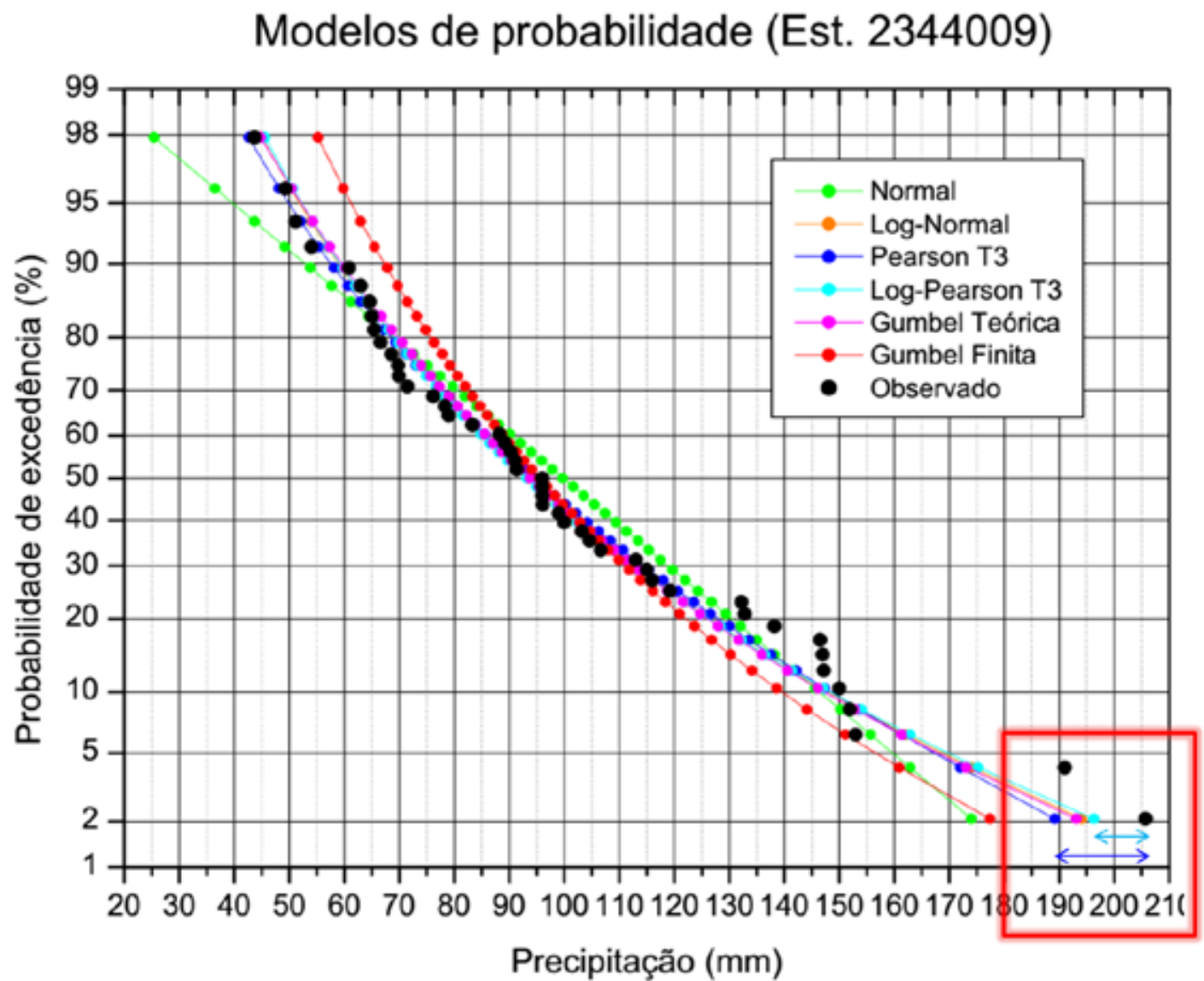

Unindo as informações mencionadas anteriormente, escolheu-se para cada estação a distribuição que representou melhor o comportamento das precipitações extremas, ou seja, às menores probabilidades de excedência; assim, indica-se a seguir a distribuição escolhida para cada caso. Para as estações $3,4,5,6,7,8,10,11,12,16,17$, $18,19,20,21,23,24$, e 25 , foi escolhida a distri- buição Gumbel Finita. Para as estações 13 e 22, foi escolhida a distribuição Pearson tipo III e para as estações 9 e 14, foi escolhida a log-Pearson tipo III. Como já é muito preconizado na literatura, Gumbel Finita foi a distribuição que mais se mostrou coerente com valores extremos.

As Tabela 4 e 5 condensam os valores dos parâmetros obtidos para cada estação. 
Tabela 4: Valores dos parâmetros K, c, m e n para durações entre 5 e 60 minutos.

\begin{tabular}{|c|c|c|c|c|c|c|}
\hline Estação & $K$ & c & m & $\mathbf{n}$ & $\begin{array}{c}\text { Média do } \\
\text { E (\%) }\end{array}$ & \# Anos \\
\hline 3 & 418,4148 & 0,1233 & 7,2369 & 0,6454 & 1,58 & 24 \\
\hline 4 & 456,1252 & 0,1362 & 7,2374 & 0,6454 & 1,86 & 38 \\
\hline 5 & 431,3320 & 0,1140 & 7,2372 & 0,6454 & 1,40 & 24 \\
\hline 6 & 449,7631 & 0,1485 & 7,2370 & 0,6454 & 2,15 & 43 \\
\hline 7 & 442,6182 & 0,1515 & 7,2370 & 0,6454 & 2,23 & 27 \\
\hline 8 & 547,8592 & 0,1861 & 7,2370 & 0,6454 & 3,24 & 56 \\
\hline 9 & 489,8050 & 0,1647 & 7,2371 & 0,6454 & 3,01 & 29 \\
\hline 10 & 544,6848 & 0,1817 & 7,2371 & 0,6454 & 3,10 & 20 \\
\hline 11 & 480,3398 & 0,1662 & 7,2369 & 0,6454 & 2,62 & 43 \\
\hline 12 & 438,3100 & 0,1728 & 7,2371 & 0,6454 & 2,82 & 24 \\
\hline 13 & 514,3748 & 0,1049 & 7,2372 & 0,6454 & 2,91 & 29 \\
\hline 14 & 665,9877 & 0,1951 & 7,2369 & 0,6454 & 3,38 & 47 \\
\hline 16 & 562,4264 & 0,2012 & 7,2371 & 0,6454 & 3,78 & 38 \\
\hline 17 & 451,6343 & 0,1614 & 7,2371 & 0,6454 & 2,49 & 29 \\
\hline 18 & 445,6021 & 0,1678 & 7,2369 & 0,6454 & 2,67 & 22 \\
\hline 19 & 479,1035 & 0,1800 & 7,2368 & 0,6454 & 3,04 & 29 \\
\hline 21 & 477,1492 & 0,1748 & 7,2371 & 0,6454 & 2,88 & 19 \\
\hline 22 & 551,1931 & 0,0941 & 7,2370 & 0,6454 & 2,49 & 33 \\
\hline 23 & 424,0091 & 0,1522 & 7,2370 & 0,6454 & 2,25 & 42 \\
\hline 24 & 382,4623 & 0,2323 & 7,2372 & 0,6454 & 5,13 & 49 \\
\hline 25 & 482,3472 & 0,1775 & 7,2370 & 0,6454 & 2,96 & 27 \\
\hline Média & 482,6448 & 0,1613 & 7,2371 & 0,6454 & 2,76 & - \\
\hline Desvio Padrão & 63,9554 & 0,0333 & 0,0001 & 0,0000 & 0,80 & - \\
\hline
\end{tabular}

Tabela 5: Valores dos parâmetros K, c, m e n para durações entre 60 e 1.440 minutos.

\begin{tabular}{|c|c|c|c|c|c|c|}
\hline Estação & $\mathbf{K}$ & c & m & $\mathbf{n}$ & $\begin{array}{c}\text { Média do } \\
\text { E (\%) }\end{array}$ & \# Anos \\
\hline 3 & 595,3278 & 0,1233 & 5,4270 & 0,7356 & 1,91 & 24 \\
\hline 4 & 648,8693 & 0,1362 & 5,4245 & 0,7355 & 2,15 & 38 \\
\hline 5 & 613,6655 & 0,1140 & 5,4265 & 0,7356 & 1,75 & 24 \\
\hline 6 & 639,8752 & 0,1485 & 5,4248 & 0,7355 & 2,41 & 43 \\
\hline 7 & 629,6811 & 0,1515 & 5,4240 & 0,7355 & 2,48 & 27 \\
\hline 8 & 779,3919 & 0,1861 & 5,4235 & 0,7355 & 3,40 & 56 \\
\hline 9 & 696,8345 & 0,1647 & 5,4253 & 0,7355 & 3,17 & 29 \\
\hline 10 & 774,8959 & 0,1817 & 5,4251 & 0,7355 & 3,26 & 20 \\
\hline 11 & 683,3669 & 0,1662 & 5,4237 & 0,7355 & 2,84 & 43 \\
\hline 12 & 623,5744 & 0,1728 & 5,4257 & 0,7355 & 3,01 & 24 \\
\hline 13 & 731,7609 & 0,1049 & 5,4242 & 0,7355 & 3,05 & 29 \\
\hline 14 & 947,4877 & 0,1951 & 5,4247 & 0,7355 & 3,52 & 47 \\
\hline 16 & 800,1231 & 0,2012 & 5,4244 & 0,7355 & 3,91 & 38 \\
\hline 17 & 642,5354 & 0,1614 & 5,4254 & 0,7356 & 2,72 & 29 \\
\hline 18 & 633,9999 & 0,1678 & 5,4267 & 0,7356 & 2,88 & 22 \\
\hline 19 & 681,6267 & 0,1800 & 5,4250 & 0,7355 & 3,21 & 29 \\
\hline 21 & 678,8239 & 0,1748 & 5,4252 & 0,7355 & 3,07 & 19 \\
\hline 22 & 784,2335 & 0,0941 & 5,4266 & 0,7356 & 2,68 & 33 \\
\hline 23 & 603,2174 & 0,1522 & 5,4247 & 0,7355 & 2,50 & 42 \\
\hline 24 & 544,0571 & 0,2323 & 5,4263 & 0,7355 & 5,24 & 49 \\
\hline 25 & 686,1972 & 0,1775 & 5,4238 & 0,7355 & 3,14 & 27 \\
\hline Média & 686,6450 & 0,1613 & 5,4251 & 0,7355 & 2,97 & - \\
\hline Desvio Padrão & 90,9882 & 0,0333 & 0,0010 & 0,0000 & 0,74 & - \\
\hline
\end{tabular}

Os erros médios obtidos entre os valores calculados e os que deram origem às curvas, nos dois casos, não superaram $3 \%$, demonstrando que os parâmetros foram bem ajustados. O maior erro foi na estação 24, que não logrou um bom ajuste aos modelos probabilísticos. 


\section{DISCUSSÕES}

Os valores mais altos do $\mathrm{P}$-valor para cada uma das distribuições foram todos para a estação 24. Esse fato pode ser explicado analisando a Figura 1 para a referida estação, que demonstra claramente que há dois regimes de chuva presentes nos dados da série daquela estação, muito possivelmente por ela ter sido movida de lugar, ou por mudanças na condição do pluviômetro (como altura e/ou surgimento de obstáculos ao redor da estação como vegetação crescente por exemplo). Por conseguinte, para dois regimes de chuvas distintos sugere-se futuramente trabalhar com distribuições bimodais.

As séries foram divididas em durações de 5 até 60 minutos e depois de 60 até 1.440 minutos, pois há uma diferença de tendência nas intensidades das chuvas de pequena e grande duração. Isso se confirma pelo fato de o fator $\mathrm{K}$ mudar drasticamente comparando-se os dois casos.

Devido ao fato de a maioria das séries pluviométricas brasileiras não possuir mais de 100 anos (período de retorno para macrodrenagem) de dados, utiliza-se frequentemente a extrapolação das mesmas por meio de modelos de distribuição de probabilidades. Para fazer a escolha do modelo, o teste K-S é comumente utilizado, de modo a escolher aquela que represente melhor os registros históricos. Os estudos de inundações, porém, têm por objetivo determinar probabilidades de excedência que representam eventos extremos. Em outras palavras, as menores probabilidades de excedência são as probabilidades que deveriam nortear os critérios de aderência dos dados a uma distribuição teórica. Dessa forma, a comparação entre valores de $D_{\text {máx }}$ e de $\mathrm{P}$-valores de séries inteiras pode levar a escolhas inadequadas, pois uma distribuição com $D_{\text {máx }}$ pequenos para grandes precipitações é preferível a uma distribuição que tenha um $D_{\text {máx }}$ global menor, mas que tenha valores de $D$ maiores para as precipitações extremas. Assim sendo, para essa finalidade específica, o teste de aderência seria mais representativo se a série histórica fosse muito grande, com 80, 90 anos por exemplo, mas fossem utilizados apenas os 20 ou 30 maiores valores de chuvas diárias, compondo-se assim uma subsérie a ser analisada separadamente.

\section{CONCLUSÕES}

Este trabalho apresentou uma análise estatística de dados de chuvas do município de Cunha e municípios vizinhos. Os melhores resultados de $\mathrm{P}$-valor foram obtidos para as distribuições da família gama, Pearson tipo III e log-Pearson tipo III, o que condiz com a literatura acerca do bom desempenho das mesmas para hidrologia. Evidencia-se, porém, a importância da análise visual das séries históricas pluviométricas quando se trata de analisar eventos extremos de máximas.

A comparação das intensidades estimadas com os parâmetros ajustados neste trabalho com os valores observados disponíveis para as estações apresentou uma diferença inferior a $3 \%$, evidenciando o bom ajuste da equação.

Completa-se ainda que, comparando-se por este método, as IDFs de 5 até 60 minutos e de 60 até 1.440 minutos, obtém-se que o parâmetro $\mathrm{K}$ é proporcional pelo fator de 1,42 aproximadamente, o parâmetro $m$ por um fator em torno de 0,75 e o parâmetro $\mathrm{n}$ por cerca de 1,14 , enquanto o parâmetro c é igual para ambos os casos.

\section{REFERÊNCIAS}

BACK, A. J. Relações Intensidade-Duração-Frequência de chuvas intensas de Chapecó, Estado de Santa Catarina. Maringá, v. 28, n. 4, p. 575-581, Out./Dez., 2006.

BERTONI, J. C.; TUCCI, C. E. Precipitação. In: TUCCI, C.E.M. (Organizador). Hidrologia Ciência e aplicação. Porto Alegre: Editora da Universidade, 2015. cap. 5, p. 177-241.

CETESB - COMPANHIA DE TECNOLOGIA DE SANEAMENTO AMBIENTAL. Drenagem Urbana: manual de projeto. São Paulo. 1980. 476p. 
CHOW, V. T. Section 8-I. Statistical and probability analysis of hydrologic data. Part I - Frequency Analysis. In: Handbook of Applied Hydrology. McGraw- Hill. USA. p. 8.1-8.42, 1964.

GRUBBS, F.; BECK G. "Extension of Sample Sizes and Percentage Points for Significance Tests of Outlying Observations", Technometrics, v. 14, n. 4, p. 847-854, 1972.
HIDROWEB. Sistema de informações hidrológicas. 2015. Disponível em: <http://hidroweb.ana.gov.br/>. Acesso em: 20 Ago. 2015.

NAGHETTINI, M.; PINTO, E. J. de A. Hidrologia Estatística. Belo Horizonte: CPRM, 2007. 552 p.

PRESS, W. H.; TEUKOLSKY, S. A.; VETTERLING, W. T.; FLANNERY, B. P. Numerical recipes - The Art of Scientific Computing. 3. ed. Cambridge University Press, New York. 1235 p. 2007. 